\title{
LARGE DERIVATIVES, BACKWARD CONTRACTION AND INVARIANT DENSITIES FOR INTERVAL MAPS
}

\author{
HENK BRUIN; JUAN RIVERA-LETELIER; \\ WEIXIAO SHEN; SEBASTIAN VAN STRIEN
}

\begin{abstract}
In this paper, we study the dynamics of a smooth multimodal interval map $f$ with non-flat critical points and all periodic points hyperbolic repelling. Assuming that $\left|\left(f^{n}\right)^{\prime}(f(c))\right| \rightarrow \infty$ as $n \rightarrow \infty$ holds for all critical points $c$, we show that $f$ satisfies the so-called backward contracting property with an arbitrarily large constant, and that $f$ has an invariant probability $\mu$ which is absolutely continuous with respect to the Lebesgue measure and the density of $\mu$ belongs to $L^{p}$ for all $p<\ell_{\max } /\left(\ell_{\max }-1\right)$, where $\ell_{\max }$ denotes the maximal critical order of $f$. In the appendix, we prove that various growth conditions on the derivatives along the critical orbits imply stronger backward contraction.
\end{abstract}

\section{INTRODUCTION}

The concept of absolutely continuous invariant measures plays an important role in studying the chaotic behavior of non-uniformly hyperbolic dynamical systems. In the area of interval dynamics, various conditions have been shown to guarantee the existence of an invariant probability which is absolutely continuous with respect to Lebesgue measure (acip). In $[\mathbf{M}]$, it was proved that an acip exists for an S-multimodal map without periodic attractors or recurrent critical points. In $[\mathbf{C E}]$, it was proved that an S-unimodal map $f$ satisfying the following condition (the Collet-Eckmann condition) has an acip:

$$
(C E) \quad \liminf _{n \rightarrow \infty} \frac{\log \left|\left(f^{n}\right)^{\prime}(f(c))\right|}{n}>0,
$$

where $c$ denotes the critical point of $f$. In [NS2], the following summability condition (the Nowicki-van Strien condition) was shown to imply the existence of an acip for an S-unimodal map:

$$
\sum_{n=0}^{\infty} \frac{1}{\left|\left(f^{n}\right)^{\prime}(f(c))\right|^{1 / \ell}}<\infty
$$

where $\ell$ is the order of the critical point. Moreover, it was proved that the density of the acip with respect to Lebesgue measure belongs to $L^{p}$ for all $p<\ell /(\ell-1)$. (Note that this regularity is the best possible since the density is never $L^{\ell /(\ell-1)}$.) Later on, this result was extended to the multimodal setting in $[\mathbf{B S}]$.

Date: January 23th, 2007.

2000 Mathematics Subject Classification. 37E05, 37C40.

For contact: Fax +86-551-3601005, Email wxshen@ustc.edu.cn; more in the last page. 
In $[\mathbf{B S S}]$, we found that for S-unimodal maps, these conditions are far too restrictive for the existence of an acip. In fact, there exists a constant $C>0$ depending on the critical order such that

$$
\liminf _{n \rightarrow+\infty}\left|\left(f^{n}\right)^{\prime}(f(c))\right| \geq C
$$

implies the existence of an acip. In this paper we shall extend this last result to the multimodal setting.

Main Theorem. Let $f:[0,1] \rightarrow[0,1]$ be a $C^{3}$ multimodal interval map with non-flat critical points and with all periodic points hyperbolic repelling. Assume that for each critical point c, we have

$$
\lim _{n \rightarrow+\infty}\left|\left(f^{n}\right)^{\prime}(f(c))\right|=\infty,
$$

then $f$ has an acip $\mu$ whose density with respect to the Lebesgue measure belongs to $L^{p}$ for all $1 \leq p<\ell_{\max } /\left(\ell_{\max }-1\right)$, where $m$ denotes the Lebesgue measure and $\ell_{\max }$ is the maximum of the orders of the critical points.

Here, as usual, by saying that $f$ is of class $C^{3}$ with non-flat critical points, we mean that $f$ is $C^{1}$ everywhere and satisfies the following:

- $f$ is $C^{3}$ outside $\operatorname{Crit}(f)=\left\{x \in[0,1]: f^{\prime}(x)=0\right\}$;

- for each $c \in \operatorname{Crit}(f)$, there exists a number $\ell_{c}>1$ (called the order of $c$ ) and $C^{3}$ diffeomorphisms $\phi, \psi$ of $\mathbb{R}$ with $\phi(c)=\psi(f(c))=0$ such that

$$
|\psi \circ f(x)|=|\phi(x)|^{\ell_{c}}
$$

holds in a neighborhood of $c$.

We shall use $\mathcal{A}$ to denote the class of all $C^{3}$ interval maps with non-flat critical points and with all periodic points hyperbolic repelling.

In order to state a more precise version of our main theorem, we need to introduce more notation. For a fixed positive integer $N$ and a positive number $\ell_{\max }>1$, denote by $\mathcal{A}\left(N, \ell_{\max }\right)$ the collection of all maps $f \in \mathcal{A}$ having exactly $N$ critical points, and for which the maximum of the orders of the critical points is $\ell_{\max }$. For $K>0$ denote by $\mathcal{A}\left(N, \ell_{\max }, K\right)$ the class of all maps $f \in \mathcal{A}\left(N, \ell_{\max }\right)$ for which the following holds:

(*) there exists a neighborhood $\mathcal{V}$ of $f($ Crit $(f))$ such that if $f^{n}(f(c)) \in \mathcal{V}$ for some critical point $c \in \operatorname{Crit}(f)$, then

$$
\left|D f^{n}(f(c))\right|>K \text {. }
$$

We shall actually prove the following Main Theorem', which clearly implies the Main Theorem.

Main Theorem'. Given a positive integer $N$, real numbers $\ell_{\max }>1$ and $p \in\left[1, \ell_{\max } /\left(\ell_{\max }-1\right)\right)$, there exists a constant $K=K\left(N, \ell_{\max }, p\right)$ such that if $f \in \mathcal{A}\left(N, \ell_{\max }, K\right)$, then $f$ has an acip $\mu$ with $d \mu / d m \in L^{p}$, where $m$ denotes the Lebesgue measure. 
The result of the density is new even in the unimodal setting. We note that the proof in this paper is quite different from and significantly simpler than that of $[\mathbf{B S S}]$. In fact, the proof follows much closer the proof in [NS2] but is simpler; the main reason for this is that we use here the notion of nice intervals which significantly simplifies the proof of formula (8) compared to the corresponding inductive statement in [NS2].

It is probably difficult to improve these results. It is not possible to give a topological condition equivalent to the existence of acip since the last property is not a topological invariant, not even a quasisymmetric invariant, see [B1]. Moreover, although no invariant density needs to exist if $\left|D f^{n}(f(c))\right|$ remains small [BKNS], $\liminf _{n \rightarrow+\infty}\left|D f^{n}(f(c))\right|=0$ does not rule out the existence of acip [B2].

Clearly, the condition $(*)$ is a $C^{1}$ invariant in the unimodal case, i.e., if for a given $K$ two unimodal maps $f, g \in \mathcal{A}$ are $C^{1}$ conjugate, and $f$ satisfies $(*)$, then so does $g$. However, for multimodal maps, this is not the case. So our Main Theorem' applies to a slightly more general case, i.e., it holds for maps $g \in \mathcal{A}$ which is $C^{1}$ conjugate to a map $f$ satisfying the requirements in the Main Theorem'.

To prove the Main Theorem', we shall use the following terminology, which was first introduced in $[\mathbf{R}]$. Let $C V=C V(f)=f(\operatorname{Crit}(f))$ be the set of critical values of $f$. We say that $f$ satisfies the backward contracting property with constant $r\left(B C(r)\right.$ in short) if the following holds: there exists $\varepsilon_{0}>0$ such that for each $\varepsilon<\varepsilon_{0}$, and any component $W$ of $f^{-s}(B(C V, r \varepsilon)), s \geq 1$,

$$
\operatorname{dist}(W, C V)<\varepsilon \text { implies }|W|<\varepsilon .
$$

We say that $f$ satisfies $B C(\infty)$ if it satisfies $B C(r)$ for all $r>1$. Clearly, for any $r>1$, the property $B C(r)$ implies that $f$ has no critical relation, i.e., no critical point is mapped into the critical set under forward iteration.

The proof of the Main Theorem' breaks into the following two propositions.

Proposition 1. For real number $\ell_{\max }>1$ and $r>1$, there exists $K=$ $K\left(r, \ell_{\max }\right)$ such that if $f$ is a map in the class $\mathcal{A}\left(N, \ell_{\max }, K\right)$ for some $N \geq 1$, then it satisfies the property $B C(r)$.

We shall provide two proofs of this proposition. The first one is given in Sect. 2 as a consequence of Lemma 2 and Proposition 4. The second one is given in the appendix, see Theorem 1 . The reason that $K$ depends only on $f$ through $N$ and $\ell_{\max }$, is that all constants related to $f$ (such as those related to non-flatness) vanish when looking at sufficiently small scales.

Proposition 2. For any positive integer $N$, real number $\ell_{\max }>1$ and $\kappa \in(0,1)$, there exists $r=r\left(N, \ell_{\max }, \kappa\right)$ such that if $f \in \mathcal{A}\left(N, \ell_{\max }\right)$ satisfies the $B C(r)$ property then there exists a constant $M$ such that for every Borel set $A$ we have

$$
\left|f^{-n}(A)\right| \leq M|f A|^{\kappa / \ell_{\max }}
$$


It follows from the inequality (3) that any weak limit $\mu$ of

$$
\frac{1}{n} \sum_{i=0}^{n-1}\left(f^{i}\right)_{*} m
$$

is an acip of $f$. By the argument above Theorem $\mathrm{A}$ in [NS2], the density of $\mu$ with respect to $m$ is in $L^{p}$, where $p \rightarrow \ell_{\max } /\left(\ell_{\max }-1\right)$ as $\kappa \rightarrow 1$.

The proof of Proposition 2 is given in Sect. 3 and 4.

In the Appendix we state and prove a result which is somewhat related to the proof of Proposition 1. It shows that various growth conditions of the derivatives along the critical orbits implies stronger backward contraction.

Remark. If $f$ is unimodal, then the acip is ergodic and unique. A general multimodal interval map may have more than one acips. For a map satisfying $B C(r)$ for a large $r$, the estimate provided by Proposition 2 rules out the existence of Cantor attractors. Thus any compact forward invariant set of positive measure contains a cycle of periodic intervals [SV], each supporting an acip.

Notation. Unless otherwise stated, $X=[0,1]$ and $f: X \rightarrow X$ is a map in the class $\mathcal{A}$. We will assume, without loss of generality, that $f(\partial X) \subset \partial X$ and that $f^{\prime}(x) \neq 0$ for $x \in \partial X$.

If $J$ is an interval and $\lambda>0$, we use $\lambda J$ to denote the concentric open interval which has length $\lambda|J|$. We say that $J$ is $\lambda$-well inside another interval $I$ or that $I$ contains the $\lambda$-scaled neighborhood of $J$, if $I \supset(1+2 \lambda) J$.

\section{REAL BOUNDS}

We shall use the following result throughout our analysis.

Proposition 3. Let $f \in \mathcal{A}$, let $s \geq 1$ be an integer and let $T=(a, b)$ be an interval. Assume that $f^{s} \mid T$ is a diffeomorphism onto its image and that $\left|f^{s}(T)\right|$ is sufficiently small. Then

(i) (the Minimum Principle) for every $x \in T$,

$$
\left|\left(f^{s}\right)^{\prime}(x)\right| \geq 0.9 \min \left(\left|\left(f^{s}\right)^{\prime}(a)\right|,\left|\left(f^{s}\right)^{\prime}(b)\right|\right) ;
$$

(ii) (the One-sided Koebe Principle) Let $x \in T$ be such that $\mid f^{s}(a)-$ $f^{s}(x)|\geq \tau| f^{s}(x)-f^{s}(b) \mid$. Then

$$
\left|\left(f^{s}\right)^{\prime}(x)\right| \geq 0.9\left(\frac{\tau}{1+\tau}\right)^{2}\left|\left(f^{s}\right)^{\prime}(b)\right|
$$

(iii) (the Koebe Principle) If $J$ is a subinterval of $T$ such that $f^{s}(J)$ is $\tau$-well inside $f^{s}(T)$, then for any $x, y \in J$,

$$
0.9\left(\frac{\tau}{1+\tau}\right)^{2} \leq \frac{\left|\left(f^{s}\right)^{\prime}(x)\right|}{\left|\left(f^{s}\right)^{\prime}(y)\right|} \leq \frac{1}{0.9}\left(\frac{1+\tau}{\tau}\right)^{2}
$$

(iv) (the Macroscopic Koebe Principle) If $J$ is a subinterval of $T$ such that $f^{s}(J)$ is $\tau$-well inside $f^{s}(T)$, then $J$ is $\tau^{\prime}$-well inside $T$, where $\tau^{\prime}=0.9 \tau^{2} /(1+2 \tau)$. 
Proof. If $f$ has negative Schwarzian derivative, then so does $f^{s} \mid T$. In this case, it is well-known that the statements hold with 0.9 being replaced by 1 (and without the assumption that $f^{s}(T)$ has a small length), see for example [MS]. For the general case, we first note that $\left|f^{s}(T)\right|$ small implies that $|T|$ is small as well since $f$ has no wandering interval, see [MS]. Then we apply a theorem of Graczyk and Sands [GS] which states that any $f \in \mathcal{A}$ is realanalytically conjugate to a map with negative Schwarzian derivative.

Remark. An alternative to prove the proposition - again for maps $f$ without negative Schwarzian derivative - is by using Theorem C of [SV].

2.1. Backward contraction. A sequence of open intervals $\left\{G_{j}\right\}_{j=0}^{s}$ is called a chain if for each $0 \leq j<s, G_{j}$ is a component of $f^{-1}\left(G_{j+1}\right)$. The order of the chain is defined to be the number of $j$ 's with $0 \leq j<s$ and such that $G_{j}$ contains a critical point.

For each critical point $c$ and $\varepsilon>0$, let $\hat{B}_{\varepsilon}(c)$ be the connected component of $f^{-1}((f(c)-\varepsilon, f(c)+\varepsilon))$ containing $c$. Moreover, let

$$
\hat{B}_{\varepsilon}=\bigcup_{c \in \operatorname{Crit}(f)} \hat{B}_{\varepsilon}(c) .
$$

Note that provided that $\varepsilon$ is small enough,

$$
\hat{B}_{r \varepsilon}(c) \approx r^{1 / \ell_{c}} \hat{B}_{\varepsilon}(c) \text {. }
$$

Lemma 1. For any $\rho>0$ and $\ell_{\max }>1$, there exists $K>1$, and for each $f \in \bigcup_{N=1}^{\infty} \mathcal{A}\left(N, \ell_{\max }, K\right)$ there exists $\varepsilon_{0}>0$ with the following property. Let $c, c^{\prime} \in \operatorname{Crit}(f)$ and $\varepsilon \in\left(0, \varepsilon_{0}\right)$. If $f^{s}(c) \in \hat{B}_{\varepsilon}\left(c^{\prime}\right)$ for some $s \geq 1$, and if $J$ is the component of $f^{-s}\left(\hat{B}_{\varepsilon}\left(c^{\prime}\right)\right)$ containing $c$, then

$$
J \subset \hat{B}_{\rho \varepsilon}(c) \text {. }
$$

Proof. We may assume that $\rho \in(0,1)$. Consider the chains $\left\{G_{j}\right\}_{j=0}^{s}$ and $\left\{H_{j}\right\}_{j=0}^{s}$ with $G_{s}=\hat{B}_{2^{\ell} c^{\prime} \varepsilon}\left(c^{\prime}\right) \supset H_{s}=\hat{B}_{\varepsilon}\left(c^{\prime}\right)$ and $G_{0} \supset H_{0}=J$.

Let $s_{1}<s$ be maximal such that $G_{s_{1}}$ contains a critical point $c_{1}$. Let $H_{s_{1}+1}^{\prime}$ be the convex hull of $H_{s_{1}+1} \cup\left\{f\left(c_{1}\right)\right\}$.

Claim. Provided that $\varepsilon$ is small enough and that $K$ is large enough, we have

$$
H_{s_{1}} \subset \hat{B}_{\rho \varepsilon}\left(c_{1}\right)
$$

In fact, since

$$
f^{s-s_{1}-1}: G_{s_{1}+1} \rightarrow G_{s}
$$

is a diffeomorphism with $\left|G_{s}\right|$ small, it follows from the One-sided Koebe Principle (Proposition 3 (ii)) that for each $x \in H_{s_{1}+1}^{\prime}$, we have

$$
\left|D f^{s-s_{1}-1}(x)\right| \geq C\left|D f^{s-s_{1}-1}\left(f\left(c_{1}\right)\right)\right|,
$$

where $C$ is a universal constant. Provided that $\varepsilon$ is small enough, we have

$$
\left|D f^{s-s_{1}}\left(f\left(c_{1}\right)\right)\right| \geq K
$$


by the hypothesis. Moreover, by non-flatness of the critical points, we have

$$
\left|D f\left(f^{s-s_{1}}\left(c_{1}\right)\right)\right| \leq 2 \ell_{c} \frac{\left|f G_{s}\right|}{\left|G_{s}\right|} .
$$

Thus

$$
\left|D f^{s-s_{1}-1}\left(f\left(c_{1}\right)\right)\right|=\frac{\left|D f^{s-s_{1}}\left(f\left(c_{1}\right)\right)\right|}{\left|D f\left(f^{s-s_{1}}\left(c_{1}\right)\right)\right|} \geq K C_{1} \frac{\left|G_{s}\right|}{\left|f G_{s}\right|},
$$

where $C_{1}=1 / 2 \ell_{c}$. This, equation (5) and the mean value theorem imply

$$
\frac{\left|G_{s}\right|}{\left|H_{s_{1}+1}^{\prime}\right|} \geq C C_{1} K \frac{\left|G_{s}\right|}{\left|f G_{s}\right|},
$$

which implies that

$$
\left|H_{s_{1}+1}^{\prime}\right| \leq \rho \varepsilon
$$

provided that $K$ is sufficiently large. The claim follows.

If $s_{1}=0$ then the proof of the lemma is completed. For the general case, the lemma follows by an easy induction on $s$.

Let us say that $f$ satisfies the property $B C^{*}(r)$ if the following holds: for any $\varepsilon>0$ small enough, $c, c^{\prime} \in \operatorname{Crit}(f)$, and $s \geq 1$, if $f^{s}(c) \in \hat{B}_{r \varepsilon}\left(c^{\prime}\right)$ and $J$ is the component of $f^{-s}\left(\hat{B}_{r \varepsilon}\left(c^{\prime}\right)\right)$ which contains $c$, then $J \subset \hat{B}_{\varepsilon}(c)$. (So the difference with the property $B C(r)$ is that in equation (2), the assumption $\operatorname{dist}(W, C V)<\varepsilon$ is replaced by $W \cap C V \neq \emptyset$.)

The above lemma can be reformulated as

Proposition 4. For any $\ell_{\max }>1$ and $r>1$ there exists $K \geq 1$ such that each $f \in \bigcup_{N=1}^{\infty} \mathcal{A}\left(N, \ell_{\max }, K\right)$ satisfies the property $B C^{*}(r)$.

The property $B C^{*}(r)$ is closely related to $B C(r)$. Clearly the latter implies the former. The other direction is shown in the following proposition.

Lemma 2. For any $f \in \mathcal{A}, B C^{*}\left(8^{\ell_{\max }} r\right)$ implies $B C(r)$, where $\ell_{\max }$ is the maximum of the order of the critical points of $f$.

Proof. Let $\varepsilon>0$ be a small constant. Then for all $c \in \operatorname{Crit}(f), \hat{B}_{8^{\ell \max r \varepsilon}}(c)$ contains the 3 -scaled neighborhood of $\hat{B}_{r \varepsilon}(c)$.

Let $c, c^{\prime} \in \operatorname{Crit}(f)$ and $x \in \hat{B}_{\varepsilon}(c)$. Let $s \geq 1$ be such that $f^{s}(x) \in \hat{B}_{r \varepsilon}\left(c^{\prime}\right)$ and let $J_{k}$ be the component of $f^{-(s-k)}\left(\hat{B}_{r \varepsilon}\left(c^{\prime}\right)\right)$ which contains $f^{k}(x)$. We want to show that $\left|J_{1}\right|<\varepsilon$.

Let us prove this by induction on $s$. For $s=1$ the statement is trivially true. Fix $s_{0}$ and assume that the statement holds if $s<s_{0}$. To prove the statement for $s=s_{0}$, consider the chain $\left\{G_{j}\right\}_{j=0}^{s}$ with $G_{s}=\hat{B}_{8^{\ell \max r \varepsilon}}\left(c^{\prime}\right)$ and $G_{0} \ni x$. We distinguish two cases:

Case 1. There exists $0 \leq s_{1}<s$ such that $G_{s_{1}}$ contains a critical point $c_{1}$. By the definition of the $B C^{*}$ property, it follows that $G_{s_{1}} \subset \hat{B}_{\varepsilon}\left(c_{1}\right)$. If $s_{1}=0$, then $c_{1}=c$ and $J_{0} \subset G_{0} \subset \hat{B}_{\varepsilon}(c)$. Otherwise, the statement follows by the induction hypothesis. 
Case 2. For any $0 \leq k<s, G_{k}$ contains no critical point. Then $f^{s-1}: G_{1} \rightarrow$ $G_{s}$ is a diffeomorphism. By the Macroscopic Koebe Principle (Proposition 3 (iii)), we obtain that $G_{1}$ contains the 1 -scaled neighborhood of $J_{1}$. Since $c \notin G_{0}, f(c) \notin G_{1}$ and $f(x) \in B_{\varepsilon}(f(c))$, it follows that $\left|J_{1}\right|<\varepsilon$.

Proof of Proposition 1. Combine Lemma 2 and Proposition 4.

2.2. Nice sets. An open set $V \subset[0,1]$ is called nice if for each $x \in \partial V$ and for any $k \geq 1, f^{k}(x) \notin V$.

Proposition 5. For any $f \in \mathcal{A}$ satisfying $B C(2)$ and any $\varepsilon>0$ sufficiently small, the following holds: for each $c \in C$ Crit $(f)$, there exists an open interval $V_{c}$ such that $\bigcup_{c \in \operatorname{Crit}(f)} V_{c}$ is nice and such that

$$
\hat{B}_{\varepsilon}(c) \subset V_{c} \subset \hat{B}_{2 \varepsilon}(c) .
$$

Proof. The proof follows from the following argument due to Rivera-Letelier, see Lemma 6.2 in $[\mathbf{R}]$. For $\varepsilon>0$ small, define the open set $V^{n}=\bigcup_{i=0}^{n} f^{-i}\left(\hat{B}_{\varepsilon}\right)$. Clearly $V^{\infty}$ is nice. Take $V_{c}^{n}$ to be the connected component of $V^{n}$ which contains $c$, and let $V_{c}=V_{c}^{\infty}$. It remains to show that $V_{c}^{n} \subset \hat{B}_{2 \varepsilon}(c)$ for each $n$. We do this by induction. For $n=0$ this holds by definition, so assume it holds for $n$. Consider $Z=f\left(V_{c}^{n+1}\right) \backslash B(f(c), \varepsilon)$. For $z \in Z$, there exists $m(z) \in\{0,1, \ldots, n\}$ and $c_{0}(z) \in \operatorname{Crit}(f)$ so that $f^{m(z)}(z) \in \hat{B}_{\varepsilon}\left(c_{0}(z)\right)$. Now choose $z_{0} \in Z$ so that $m_{0}=m\left(z_{0}\right)$ is minimal among $m(z)$ for points $z \in Z$ and let $\hat{c}_{0}=c_{0}(z)$. Since $f^{m_{0}}(z) \in \hat{B}_{\varepsilon}\left(\hat{c}_{0}\right)$, and since $f^{m_{0}}(Z) \subset V_{c}^{n}$, the induction hypothesis implies

$$
f^{m_{0}}(Z) \subset \hat{B}_{2 \varepsilon}\left(\hat{c}_{0}\right) .
$$

Since $f$ satisfies $B C(2)$ and $Z$ has distance $\varepsilon$ to $f(c)$, it follows that $|f(Z)|<$ $\varepsilon$ and $Z \subset \hat{B}_{2 \varepsilon}(c)$. This completes the induction step.

For $\lambda>0$ we say that a nice open set $V$ is $\lambda$-nice if for each return domain $J$ of $V$, we have $(1+2 \lambda) J \subset V$.

A nice open set $V \supset \operatorname{Crit}(f)$ will be called a puzzle neighborhood of $\operatorname{Crit}(f)$ if each component of $V$ contains exactly one critical point of $f$.

Lemma 3. For any $\lambda>0$ and real number $\ell_{\max }>1$, there exists $r>1$ such that for any $N$ and any $f \in \mathcal{A}\left(N, \ell_{\max }\right)$ which satisfies $B C(r)$ the following holds. There exists $\varepsilon_{0}>0$ such that for any $\varepsilon \in\left(0, \varepsilon_{0}\right)$ there exists a puzzle neighborhood $V=\bigcup_{c} V_{c}$ with the following properties:

- $V$ is $\lambda$-nice;

- for each $c \in \operatorname{Crit}(f)$ we have

$$
\hat{B}_{\varepsilon}(c) \subset V_{c} \subset \hat{B}_{2 \varepsilon}(c) .
$$

Proof. By Proposition 5, there exists a puzzle neighborhood $W$ of $\operatorname{Crit}(f)$ such that

$$
\hat{B}_{r \varepsilon / 2}(c) \subset W_{c} \subset \hat{B}_{r \varepsilon}(c)
$$


For each $c \in \operatorname{Crit}(f)$, let $V_{c}$ be the union of $\hat{B}_{\varepsilon}(c)$ and all return domains of $W$ which intersects $\hat{B}_{\varepsilon}(c)$. Clearly, $V=\bigcup_{c} V_{c}$ is a puzzle neighborhood of Crit $(f)$ and moreover, for each $x \in \partial V$ and $k \geq 1, f^{k}(x) \notin W$. Provided $r$ is large enough, each component of $V$ is deep inside a component of $W$. It follows that each return domain of $V$ is deep inside $V$, see for example Theorem $\mathrm{B}(2)$ in $[\mathbf{S V}]$.

If $I$ is an interval which contains a critical point and $J$ (with $J \cap$ Crit $\neq \emptyset$ ) is a unicritical pull back of $I$ then we say that $J$ is a child of $I$. So there exists $c \in \operatorname{Crit}(f), s \geq 1$ (called the transition time from $J$ to $I$ ) and an interval $\tilde{J} \ni f(c)$ such that

- $f^{s-1}$ maps $\tilde{J}$ diffeomorphically onto $I$;

- $J$ is the component of $f^{-1}(\tilde{J})$ which contains $c$.

We shall use the following lemma in the next section:

Lemma 4. Let $c, c^{\prime} \in \operatorname{Crit}(f)$, let $I \ni c$ be a $\lambda$-nice interval with $|I|$ small and let

$$
J_{1} \supsetneq J_{2} \supsetneq \cdots \supsetneq J_{m}
$$

be children of I which contain $c^{\prime}$. Then

$$
\left|f\left(J_{i}\right)\right| \leq \rho^{i-1}\left|f\left(J_{1}\right)\right|
$$

holds for all $i$, where $\rho=\rho(\lambda)>0$ is such that $\rho \rightarrow 0$ as $\lambda \rightarrow \infty$.

Proof. By definition, for each $i \geq 1$, there exists a positive integer $s_{i}$ and an interval $T_{i} \supset f J_{i}$ such that $f^{s_{i}-1}$ maps $T_{i}$ diffeomorphically onto $I$ and $J_{i}$ is the component of $f^{-1}\left(T_{i}\right)$ containing $c^{\prime}$. Note that $f^{s_{i}}\left(T_{i+1}\right)$ is contained in a return domain of $I$, hence $\lambda$-well inside $I$. By the Koebe Principle, it follows that each $T_{i+1}$ is $\lambda_{1}$-well inside $T_{i}$, where $\lambda_{1} \rightarrow \infty$ as $\lambda \rightarrow \infty$. The conclusion follows.

\section{Pull BaCK of intervals}

The goal of this section is to prove the following:

Proposition 6. For $\kappa \in(0,1), N \in \mathbb{N}$ and $\ell_{\max }>1$, there exists $r>1$ such that if $f \in \mathcal{A}\left(N, \ell_{\max }\right)$ satisfies $B C(r)$, then there exists a neighborhood $U$ of $C r i t(f)$ such that for any interval $A \subset U$ and any $n \geq 0$, the following holds:

$$
\left|f^{-n}(A)\right| \leq M|f(A)|^{\kappa / \ell_{\max }}
$$

where $M$ is a constant depending on $f$.

Let $r$ be a large constant, and assume that $f$ satisfies $B C(r)$. By Lemma 3, there exist $\lambda=\lambda\left(r, \ell_{\max }\right)$ and $\varepsilon_{0}=\varepsilon_{0}(f)>0$ small such that for any $\varepsilon<\varepsilon_{0}$ there exists a $\lambda$-nice puzzle neighborhood of Crit $(f)$ which lies in-between $\hat{B}_{\varepsilon}$ and $\hat{B}_{2 \varepsilon}$. Moreover, $\lambda \rightarrow \infty$ as $r \rightarrow \infty$. 
Set

$$
r_{1}=r^{1 / \ell_{\max }}
$$

For each $n \geq 0$ and $\delta>0$, define

$$
L_{n}(\delta)=\sup \left\{\left|f^{-m}(A)\right|: 0 \leq m \leq n, A \subset \hat{B}_{\varepsilon_{0} / r_{1}} \text { is an interval, }|f A| \leq \delta\right\} .
$$

Lemma 5. Let I be a $\lambda$-nice interval such that $\hat{B}_{\varepsilon}(c) \subset I \subset \hat{B}_{2 \varepsilon}(c)$, where $c \in \operatorname{Crit}(f)$ and $\varepsilon<\varepsilon_{0}$, and let $A$ be an interval such that

$$
A \subset \hat{B}_{\varepsilon / 2}(c) \text { and } \bar{A} \not \subset \hat{B}_{\varepsilon / r_{1}}(c) \text {. }
$$

Then for all $n \geq 1$,

$$
\left|f^{-n}(A)\right| \leq C \frac{|A|}{|I|}\left|f^{-n}(I)\right|+2 N \sum_{i=1}^{\infty} L_{n-1}\left(\rho^{i}|f A|\right),
$$

where $N=\# \operatorname{Crit}(f)$ and $C>0, \rho \in(0,1)$ are constants depending only on $r$ and $\ell_{\max }$. Moreover, $\rho \rightarrow 0$ as $r \rightarrow \infty$ (for a fixed $\ell_{\max }$ ).

Proof. Let $\mathcal{J}_{0}$ be the collection of all components $J$ of $f^{-n}(I)$ such that $f^{n}: J \rightarrow I$ is a diffeomorphism. Let $\mathcal{J}_{1}$ be the collection of all other components of $f^{-n}(I)$.

By the Koebe Principle, for all $J \in \mathcal{J}_{0}$, we have

$$
\frac{\left|f^{-n}(A) \cap J\right|}{|J|} \leq C \frac{|A|}{|I|}
$$

where $C$ is a constant depending only on $\ell_{\max }$.

For each $J \in \mathcal{J}_{1}$, there exist $n_{1}=n_{1}(J) \in\{0,1, \ldots, n-1\}$ and an interval $J^{\prime} \supset f^{n-n_{1}}(J)$ such that $J^{\prime}$ has a common endpoint with $f^{n-n_{1}}(J)$ and such that $J^{\prime}$ is a child of $I$.

Claim. There exists a constant $C>0$ depending only on $\ell_{\max }$ such that for any $J \in \mathcal{J}_{1}$, and each component $A^{\prime}$ of $f^{-n_{1}}(A) \cap J^{\prime}$, we have

$$
\left|f\left(A^{\prime}\right)\right| \leq C r_{1}^{\ell_{c}-1} \frac{\left|f J^{\prime}\right|}{|f I|}|f(A)| .
$$

In fact, by definition of a child, there exists $\tilde{J}^{\prime} \supset f\left(J^{\prime}\right)$ such that $f^{n_{1}-1}$ maps $\tilde{J}^{\prime}$ diffeomorphically onto $I$. By the One-sided Koebe Principle, there exists a constant $C$ such that

$$
\frac{\left|f A^{\prime}\right|}{\left|f J^{\prime}\right|} \leq C \frac{|A|}{\left|f^{n_{1}}\left(J^{\prime}\right)\right|}
$$

Since $A \subset I \subset \hat{B}_{2 \varepsilon}(c)$, and $A \not \subset \hat{B}_{\varepsilon / r_{1}}(c)$, we have

$$
\frac{|f I|}{|I|} \frac{|A|}{|f A|} \leq\left(2 r_{1}\right)^{\ell_{c}-1}
$$

Since $A \subset \hat{B}_{\varepsilon / 2}(c), I \supset \hat{B}_{\varepsilon}(c)$ and since $f^{n_{1}}\left(J^{\prime}\right)$ contains a component of $I \backslash A,\left|f^{n_{1}}\left(J^{\prime}\right)\right| /|I|$ is bounded away from zero. Inequality (10) follows by redefining the constant $C$. 
For any child $P$ of $I$, let $s(P)$ be the transition time from $P$ to $I$ and let $\mathcal{J}_{1}(P)$ be the collection of all elements $J \in \mathcal{J}_{1}$ with $J^{\prime}=P$. Clearly,

$$
\sum_{J \in \mathcal{J}_{1}(P)}\left|f^{-n}(A) \cap J\right|=\left|f^{-(n-s(P))}\left(f^{-s(P)}(A) \cap P\right)\right| .
$$

Since $f^{-s(P)}(A) \cap P$ has at most two components, applying (10) we obtain that

$$
\sum_{J \in \mathcal{J}_{1}(P)}\left|f^{-n}(A) \cap J\right| \leq 2 L_{n-1}\left(C r_{1}^{\ell_{c}-1} \frac{|f P|}{|f I|}|f A|\right) .
$$

For each $c^{\prime} \in \operatorname{Crit}(f)$, let

$$
P_{1}\left(c^{\prime}\right) \supsetneq P_{2}\left(c^{\prime}\right) \supsetneq \cdots
$$

be all the children of $I$ which contain $c^{\prime}$. By the $B C(r)$ property,

$$
\left|f\left(P_{1}\left(c^{\prime}\right)\right)\right| \leq 2 \varepsilon / r .
$$

By Lemma 4,

$$
\left|f\left(P_{i}\left(c^{\prime}\right)\right)\right| \subset \rho_{1}^{i-1}\left|f\left(P_{1}\left(c^{\prime}\right)\right)\right|,
$$

where $\rho_{1}$ is a constant depending on $\lambda$, and $\rho_{1} \rightarrow 0$ as $\lambda \rightarrow \infty$. So

$$
f\left(P_{i}\left(c^{\prime}\right)\right) \subset B_{2 \rho_{1}^{i-1} \varepsilon / r}\left(c^{\prime}\right) .
$$

Note that since $r$ is large, and $r_{1}=r^{1 / \ell_{\max }}$, for each $c, \quad r_{1}^{\ell_{c}-1} / r \leq r_{1}^{\ell_{\max }-1} / r$ is small. Hence

$$
\rho:=\max \left(\rho_{1}, 2 C r_{1}^{\ell_{\max }-1} / r\right)
$$

is close to zero. By (11),

$$
\sum_{J \in \mathcal{J}_{1}\left(P_{i}\left(c^{\prime}\right)\right)}\left|f^{-n}(A) \cap J\right| \leq 2 L_{n-1}\left(\rho^{i}|f A|\right) .
$$

Thus

$$
\begin{aligned}
\sum_{J \in \mathcal{J}_{1}}\left|f^{-n}(A) \cap J\right| & =\sum_{c^{\prime}} \sum_{i=1}^{\infty} \sum_{J \in \mathcal{J}_{1}\left(P_{i}\left(c^{\prime}\right)\right)}\left|f^{-n}(A) \cap J\right| \\
& \leq 2 N \sum_{i=1}^{\infty} L_{n-1}\left(\rho^{i}|f A|\right) .
\end{aligned}
$$

Combining this with (9), we obtain inequality (8).

Proof of Proposition 6 . Fix $\kappa \in(0,1)$ and write $\alpha=\kappa / \ell_{\max }$. Let

$$
M=\left(\frac{r_{1}}{\varepsilon_{0}}\right)^{\alpha} \text {. }
$$

Assume that $r$ is so large that

$$
4 C r_{1}^{(\kappa-1) / \ell_{\max }} \leq \frac{1}{2},
$$

where $C$ is as in Lemma 5 . We shall prove by induction on $n$ that the following inequality (12) holds:

$$
L_{n}(\delta) \leq M \delta^{\alpha} .
$$


The case $n=0$ is trivial. So assume that the inequality holds for all $n<n_{0}$. To show that inequality (12) is true for $n=n_{0}$, we shall prove by induction on $m$ the following:

$$
L_{n}\left(\frac{\varepsilon_{0}}{2^{m}}\right) \leq M\left(\frac{\varepsilon_{0}}{2^{m}}\right)^{\alpha} .
$$

The choice of $M$ clearly guaranteed that inequality (13) holds for $m=0$. Assuming that the inequality holds for all $m$ less than some positive integer $m_{0}$, let us prove it for $m=m_{0}$.

Let $A \subset \hat{B}_{\varepsilon_{0} / r_{1}}$ be an open interval with $|f A| \leq \varepsilon_{0} / 2^{m_{0}}$. We need to show that

$$
\left|f^{-n}(A)\right| \leq M|f A|^{\alpha}
$$

Let $\varepsilon \in\left(0, \varepsilon_{0}\right)$ be minimal such that $A \subset \hat{B}_{\varepsilon / r_{1}}(c)$ for some $c \in \operatorname{Crit}(f)$. Let $I \ni c$ be a $\lambda$-nice interval such that

$$
\hat{B}_{\varepsilon} \subset I \subset \hat{B}_{2 \varepsilon} .
$$

Then by Lemma 5, we have

$$
\left|f^{-n}(A)\right| \leq C \frac{|A|}{|I|}\left|f^{-n}(I)\right|+2 N \sum_{i \geq 1} L_{n-1}\left(\rho^{i}|f A|\right) .
$$

The second term in the right hand side is bounded from above by

$$
2 N M \sum_{i \geq 1}\left(\rho^{i}|f A|\right)^{\alpha}=2 N M|f A|^{\alpha} \frac{\rho^{\alpha}}{1-\rho^{\alpha}} \leq|f A|^{\alpha} M / 2,
$$

provided that $\rho$ is small enough. If $I \subset \hat{B}_{\varepsilon_{0} / r_{1}}$, then by the induction hypothesis,

$$
\left|f^{-n}(I)\right| \leq M|f I|^{\alpha}
$$

since $|f I| \geq \varepsilon_{0} / 2^{m_{0}-1}$. The same estimate holds in the case $I \supset \hat{B}_{\varepsilon_{0} / r_{1}}$ by the choice of $M$ since $\left|f^{-n}(I)\right| \leq 1$. Thus

$$
\left|f^{-n}(A)\right| \leq C M \frac{|A|}{|I|}|f I|^{\alpha}+\frac{M}{2}|f A|^{\alpha} .
$$

Since

$$
\frac{|A|}{|I|} \leq \frac{|f A|}{|f I|} \cdot 4 r_{1}^{1-1 / \ell_{c}}
$$

it follows that

$$
\left|f^{-n}(A)\right| \leq M\left(4 C r_{1}^{(\kappa-1) / \ell_{\max }}+\frac{1}{2}\right)|f A|^{\alpha} \leq M|f A|^{\alpha} .
$$

This completes the proof of inequality (13), hence the induction step in (12) and the proposition. 


\section{INVARIANT MEASURE AND THE DENSITY}

In this section we prove Proposition 2. So let $f$ be a multimodal interval map as in the proposition.

The strategy of the proof of (3) is to use the Minimum Principle (Proposition 3 (i)) to relate the size of the preimage of a general set to the size of preimages of intervals "at the end of branches", see Lemma 6 below. This corresponds to the "sliding argument" used in [NS2].

Take $\kappa_{1} \in(\kappa, 1)$. Assume that $f$ satisfies $B C(r)$ for a large $r$. Then by Proposition 6 , there exists $\varepsilon_{0}>0$ (small) such that for any interval $Q \subset \hat{B}_{\varepsilon_{0}}$ and any $n \geq 0$,

$$
\left|f^{-n}(Q)\right| \leq M_{0}|f Q|^{\kappa_{1} / \ell_{\max }}
$$

where $M_{0}>0$ is a constant.

We say that a sequence of open intervals $\left\{G_{i}\right\}_{i=0}^{s}$ is a quasi-chain if for each $0 \leq i<s, G_{i}$ contains a component of $f^{-1}\left(G_{i+1}\right)$. The order of the quasi-chain is defined to be the number of $i \in\{0,1, \ldots, s-1\}$ such that $G_{i}$ contains a critical point. We say that such a quasi-chain is $\lambda$-admissible if for each $0 \leq i \leq s$, either $G_{i} \cap \operatorname{Crit}(f)=\emptyset$, or $G_{i}$ is $\lambda$-nice.

By the argument in Sect. 2, choosing $\varepsilon_{0}$ smaller if necessary, we may assume that for each $j \geq 0$, there exists a $\lambda$-nice interval $V_{j, c}$ with the following property:

$$
\hat{B}_{\varepsilon_{0} / 2^{j+1}}(c) \subset V_{j, c} \subset \hat{B}_{\varepsilon_{0} / 2^{j}}(c),
$$

where $\lambda \rightarrow \infty$ as $r \rightarrow \infty$. For each interval $\tilde{G}$ which contains a critical point $c$ and with $\tilde{G} \subset V_{0, c}$, let $j$ be maximal such that $\tilde{G} \subset V_{j, c}$. We call that $V_{j, c}$ is the enlargement of $\tilde{G}$. Clearly, the enlargement of any critical interval is uniformly comparable to itself in size.

Let $V=\bigcup_{c \in \operatorname{Crit}(f)} V_{0, c}$. Fix a positive integer $n$ throughout the rest of this section. For each component $J$ of $f^{-n}(V)$, we define a $\lambda$-admissible quasi-chain $\left\{G_{i}\right\}_{i=0}^{n}$ as follows:

- $G_{s}=V$;

- Assume that $G_{i+1} \supset f^{i+1}(J)$ is defined, and let $\tilde{G}_{i}$ be the component of $f^{-1}\left(G_{i+1}\right)$ which contains $f^{i}(J)$. Then

- if $\tilde{G}_{i}$ is not critical, then $G_{i}:=\tilde{G}_{i}$;

- if $\tilde{G}_{i}$ is critical, then $G_{i}$ is the enlargement of $\tilde{G}_{i}$ defined as above.

Let $\mathcal{J}_{m}$ be the collection of all components of $f^{-n}(V)$ such that the order of the corresponding quasi-chain $\left\{G_{i}\right\}_{i=0}^{n}$ is equal to $m$. Each $J \in \mathcal{J}_{m}$ is associated with a sequence of positive integers $k_{1}, k_{2}, \ldots, k_{m}$ and critical points $c_{0}, c_{1}, \ldots, c_{m}$ as follows.

- $s_{0}=s>s_{1}>\ldots>s_{m} \geq 0$ are all the integers such that $G_{s_{j}}$ is critical, and $c_{j}$ is the critical point contained in $G_{s_{j}}$;

- $\tilde{G}_{s_{j}}$ is the $k_{j}$-th child of $G_{s_{j-1}}$ which contains $c_{j}$. 
For any $\mathbf{c}=c_{0} c_{1} \ldots c_{m} \in \operatorname{Crit}(f)^{m+1}$ and $\mathbf{k}=k_{1} k_{2} \ldots k_{m} \in \mathbb{Z}_{+}^{m}$, we use $\mathcal{J}_{\mathbf{c}}^{\mathbf{k}}$ to denote the collection of all components $J$ of $f^{-n}(V)$ with the parameters $\mathbf{c}$ and $\mathbf{k}$. Note that if $J^{\prime} \in \mathcal{J}_{\mathbf{c}}^{\mathbf{k}}$ and $\left\{G_{j}^{\prime}\right\}_{j=0}^{n}$ is the corresponding quasi-chain, then $s_{j}=s_{j}^{\prime}$ and $G_{s_{j}}=G_{s_{j}}^{\prime}$ for all $0 \leq j \leq m$. Define

$$
H_{\mathbf{c}}^{\mathbf{k}}=G_{s_{m}}
$$

and

$$
C V_{\mathbf{c}}^{\mathbf{k}}=\left\{f^{s_{0}-s_{j}}\left(c_{j}\right): 0 \leq j \leq m\right\}
$$

which has $m+1$ elements. (Recall that $f$ has no critical relation.) Notice that for each $J \in \mathcal{J}_{\mathbf{c}}^{\mathbf{k}}$, the critical values of $\left.f^{n}\right|_{J}$ are contained in $C V_{\mathbf{c}}^{\mathbf{k}}$.

Lemma 6. For any $\boldsymbol{c}, \boldsymbol{k}$, and any Borel set $A \subset V$, the following holds:

$$
\sum_{J \in \mathcal{J}_{c}^{k}}\left|f^{-n}(A) \cap J\right| \leq 3 M_{0}(m+2)|f A|^{\kappa_{1} / \ell_{\max }},
$$

where $M_{0}$ is as in (15).

Proof. Let us label the set $C V_{\mathbf{c}}^{\mathbf{k}}$ as $v_{1}<v_{2}<\ldots<v_{m}$, and let $v_{0}$ and $v_{m+2}$ be the left and right endpoints of $V_{c_{0}}$. These points divide $V_{c_{0}}$ into subintervals $Q_{k}=\left(v_{k}, v_{k+1}\right), 0 \leq k \leq m+1$. Let $A_{k}=A \cap Q_{k}$. It suffices to prove that for each $k$, the following holds:

$$
\sum_{J \in \mathcal{J}_{\mathbf{c}}^{\mathbf{k}}}\left|f^{-n}\left(A_{k}\right) \cap J\right| \leq 2 M_{0} \rho^{\kappa_{1} / \ell_{\max }}
$$

where $\rho=|f A|$. If $\left|f Q_{k}\right| \leq \rho$, then by Proposition 6 ,

$$
\sum_{J \in \mathcal{J}_{\mathbf{c}}^{\mathbf{k}}}\left|f^{-n}\left(A_{k}\right) \cap J\right| \leq\left|f^{-n} Q_{k}\right| \leq M_{0} \rho^{\kappa_{1} / \ell_{\max }} .
$$

So assume that $\left|f Q_{k}\right|>\rho$. To show inequality (17), choose $a_{k}, b_{k} \in Q_{k}$ such that

satisfies

$$
L_{k}=\left(v_{k}, a_{k}\right), \text { and } R_{k}=\left(b_{k}, v_{k+1}\right)
$$

$$
\left|f L_{k}\right|=\left|f R_{k}\right|=\left|f A_{k}\right|
$$

For each $J \in \mathcal{J}_{\mathbf{c}}^{\mathbf{k}}$ and each component $J^{\prime}$ of $f^{-n} Q_{k} \cap J, f^{n+1}: J^{\prime} \rightarrow f Q_{k}$ is a diffeomorphism. By the Minimum Principle it follows that

$$
\begin{aligned}
\left|\left(f^{n} \mid J^{\prime}\right)^{-1}\left(A_{k}\right)\right| & =\left|\left(f^{n+1} \mid J^{\prime}\right)^{-1}\left(f A_{k}\right)\right| \\
& \leq 0.9^{-1}\left(\left|\left(f^{n+1} \mid J^{\prime}\right)^{-1}\left(f L_{k}\right)\right|+\left|\left(f^{n+1} \mid J^{\prime}\right)^{-1}\left(f R_{k}\right)\right|\right) \\
& =0.9^{-1}\left(\left|f^{-n} L_{k} \cap J^{\prime}\right|+\left|f^{-n} R_{k} \cap J^{\prime}\right|\right) .
\end{aligned}
$$

Thus,

$$
\begin{aligned}
\sum_{J \in \mathcal{J}_{\mathbf{c}}^{\mathbf{k}}}\left|f^{-n}\left(A_{k}\right) \cap J\right| & \leq 0.9^{-1} \sum_{J \in \mathcal{J}_{\mathbf{c}}^{\mathbf{k}}}\left(\left|f^{-n} L_{k} \cap J\right|+\left|f^{-n} R_{k} \cap J\right|\right) \\
& \leq 0.9^{-1}\left(\left|f^{-n} L_{k}\right|+\left|f^{-n} R_{k}\right|\right) \\
& \leq 3 M_{0} \rho^{\kappa_{1} / \ell_{\max }}
\end{aligned}
$$

where in the last step we used (15). This proves (17), and hence completes the proof of the lemma. 
Applied to the case $\mathbf{c}=\{c\}, c \in \operatorname{Crit}(f)$, the lemma gives

$$
\sum_{J \in \mathcal{J}_{0}}\left|f^{-n}(A) \cap J\right| \leq 6 N M_{0}|f A|^{\kappa_{1} / \ell_{\max }} .
$$

Lemma 7. There is $\sigma>0$ depending only on $r$, such that $\sigma \rightarrow 0$ as $r \rightarrow+\infty$, and such that for each $\mathbf{c}=c_{0} c_{1} \ldots c_{m}$ and $\mathbf{k}=k_{0} k_{1} \ldots k_{m}$ the following hold:

(i)

(ii)

$$
\bigcup_{J \in \mathcal{J}_{\mathbf{c}}^{\mathbf{k}}} J \subset f^{-\left(n-s_{m}\right)}\left(H_{\mathbf{c}}^{\mathbf{k}}\right)
$$

$$
\left|f\left(H_{\mathbf{c}}^{\mathbf{k}}\right)\right| \leq \sigma^{k_{1}+k_{2}+\ldots+k_{m}}|f V|,
$$

(iii) if we put $\sigma_{1}=\sigma^{\kappa_{1} / \ell_{\max }}$, then

$$
\sum_{J \in \mathcal{J}_{\mathbf{c}}^{\mathbf{k}}}|J| \leq M_{1} \sigma_{1}^{k_{1}+k_{2}+\ldots+k_{m}}
$$

where $M_{1}=M_{0}|f V|^{\kappa_{1} / \ell_{\max }}$, and $M_{0}$ is as in (15).

Proof. The first item is clear. The second follows from Lemma 4, and $\left|\tilde{G}_{s_{i}}\right| \asymp$ $\left|G_{s_{i}}\right|$. For the third item, notice that by item (i), the left hand side of the inequality does not exceed $\left|f^{-\left(n-s_{m}\right)}\left(H_{\mathbf{c}}^{\mathbf{k}}\right)\right|$. So the inequality follows from the item (ii) of this lemma by (15).

Lemma 8. Provided that $r$ is large enough, the following holds: for any Borel set $A \subset V$ and any $n \geq 1$,

$$
\left|f^{-n}(A)\right| \leq M|f A|^{\kappa / \ell_{\max }}
$$

where $M$ is a constant.

Proof. Pick $D_{0}$ to be the maximal integer such that

$$
6(1+N)^{D_{0}+1} D_{0} \leq\left(\frac{1}{\rho}\right)^{\left(\kappa_{1}-\kappa\right) / \ell_{\max }},
$$

where $\rho=|f A|$ and $N=\# \operatorname{Crit}(f)$. For any $m \geq 1$ and $D \geq 1$, let

$$
\begin{gathered}
\mathcal{J}_{m}(D)=\left\{J \in \mathcal{J}_{m}: k_{1}(J)+k_{2}(J)+\ldots+k_{m}(J)=D\right\}, \\
\mathcal{J}(D)=\bigcup_{m=1}^{\infty} \mathcal{J}_{m}(D) .
\end{gathered}
$$

For each $1 \leq m \leq D$, the number of tuples $\mathbf{k}=k_{1} k_{2} \ldots k_{m}$ satisfying

$$
k_{1}+k_{2}+\ldots+k_{m}=D
$$

is given by the binomial coefficient $C_{D-1}^{m-1}:=\left(\begin{array}{c}D-1 \\ m-1\end{array}\right)$. Associated to such a $\mathbf{k}=k_{1} k_{2} \ldots k_{m}$, there could be at most $N^{m+1}$ corresponding choices of $\mathbf{c}=c_{0} c_{1} \ldots c_{m}$. From this and Lemma 7 it follows that

$$
\sum_{J \in \mathcal{J}(D)}|J| \leq \sum_{m=1}^{D} \sum_{J \in \mathcal{J}_{m}(D)}|J| \leq M_{1} \sum_{m=1}^{D} N^{m+1} C_{D-1}^{m-1} \sigma_{1}^{D} \leq M_{1} N \sigma_{2}^{D},
$$


where $\sigma_{2}=(N+1) \sigma_{1}$ and $M_{1}$ is as in Lemma 7 . Thus

$$
\sum_{D=D_{0}+1}^{\infty} \sum_{J \in \mathcal{J}(D)}\left|f^{-n}(A) \cap J\right| \leq \sum_{D=D_{0}+1}^{\infty} \sum_{J \in \mathcal{J}(D)}|J| \leq M_{1} \frac{N \sigma_{2}}{1-\sigma_{2}} \sigma_{2}^{D_{0}} .
$$

Provided that $r$ is large enough, $\sigma_{2}$ is close to 0 so that

$$
\sigma_{2}^{\hat{D}} \leq\left[6(1+N)^{\hat{D}+2}(\hat{D}+1)\right]^{-\tau}
$$

for any $\hat{D} \geq 1$ and where $\tau$ is so that $\tau\left(\kappa_{1}-\kappa\right)=\kappa$. This and (19) imply

$$
\sigma_{2}^{D_{0}+1} \leq \rho^{\tau\left(\kappa_{1}-\kappa\right) / \ell_{\max }}=\rho^{\kappa / \ell_{\max }} .
$$

Since $\rho=|f A|$ we get therefore

$$
\sum_{D=D_{0}+1}^{\infty} \sum_{J \in \mathcal{J}(D)}\left|f^{-n}(A) \cap J\right| \leq M_{2}|f A|^{\kappa / \ell_{\max }},
$$

where $M_{2}$ is a constant.

On the other hand, by (16), we have

$$
\begin{aligned}
\sum_{D=1}^{D_{0}} \sum_{J \in \mathcal{J}(D)}\left|f^{-n}(A) \cap J\right| & \leq \sum_{m=1}^{D_{0}} \sum_{J \in \mathcal{J}_{m}}\left|f^{-n}(A) \cap J\right| \\
& \leq \sum_{m=1}^{D_{0}} C_{D_{0}-1}^{m-1} N^{m+1} 3 M_{0}(m+2)|f A|^{\kappa_{1} / \ell_{\max }} \\
& \leq 6 M_{0}(1+N)^{D_{0}+1} D_{0}|f A|^{\kappa_{1} / \ell_{\max }} \\
& \leq M_{0}|f A|^{\kappa / \ell_{\max }},
\end{aligned}
$$

where in the last inequality we used (19) again. Combining with (18), these estimates imply the lemma.

Proof of Proposition 2. Fix $\kappa \in(0,1)$. Assume that $f$ satisfies $B C(r)$ for a large $r$. We want to show that there exists $M>0$ such that inequality (3) holds for any Borel set $A$ and any $n \geq 0$.

By Lemma 8, there exists a neighborhood $V$ of Crit $(f)$ such that the inequality holds when $A \subset V$.

The general case follows by Mãné's theorem, which asserts that $f \mid(X \backslash V)$ is uniformly expanding, i.e., there exists $C>0$ and $\gamma \in(0,1)$ such that for any $x \in X$ and $k \geq 1$, if $x, f(x), \ldots, f^{k-1}(x) \notin V$ then $\left|\left(f^{k}\right)^{\prime}(x)\right| \geq C \gamma^{-k}$. It follows that for any Borel set $A$ and $m \geq 1$,

$$
A_{m}:=\left\{x \in X: x, f(x), \ldots, f^{m-1}(x) \notin V, f^{m}(x) \in A\right\}
$$

has length $C_{1} \gamma_{1}^{m}|A|$, where $C_{1}>0$ and $\gamma_{1} \in(0,1)$ are constants.

Set $Q_{n}=A_{n}$ and for $0 \leq m<n$, set

$$
Q_{m}:=\left\{x: f^{m}(x) \in V \text { and } f^{m+1}(x) \in A_{n-m-1}\right\} .
$$

Clearly,

$$
f^{-n}(A)=\bigcup_{m=0}^{n} Q_{m}
$$


By the argument in the previous paragraph,

$$
\left|Q_{n}\right| \leq\left|A_{n}\right| \leq C_{1} \gamma_{1}^{n}|A|
$$

and for $0 \leq m<n$, since $f^{m}\left(Q_{m}\right) \subset V \cap f^{-1} A_{n-m-1}$, we have

$$
\begin{aligned}
\left|Q_{m}\right| & \leq\left|f^{-m}\left(f^{m}\left(Q_{m}\right)\right)\right| \leq\left|f^{-m}\left(V \cap f^{-1} A_{n-m-1}\right)\right| \\
& \leq M\left|A_{n-m-1}\right|^{\alpha} \leq M^{\prime} \gamma_{2}^{n-m}|A|^{\alpha},
\end{aligned}
$$

where the third inequality follows by Lemma 8 , and $M^{\prime}>0$ and $\gamma_{2} \in(0,1)$ are constants. Inequality (3) follows by redefining $M$.

\section{Appendix: Growth OF DERIVATIVES AND BACKWARD CONTRACTION}

Let $f: X \rightarrow X$ be a map in the class $\mathcal{A}$, i.e., $f$ is $C^{3}$ with non-flat critical points and all periodic points hyperbolic repelling. Let $C V=C V(f)=$ $f(\operatorname{Crit}(f))$. Given $\delta^{\prime}>\delta>0$ we will say that $f$ is $\left(\delta, \delta^{\prime}\right)$-backward contracting if for every critical point $c$ of $f$, every $n \geq 1$, and every connected component $W$ of $f^{-n}\left(\hat{B}_{\delta^{\prime}}(c)\right)$ we have that

$$
\operatorname{dist}(W, C V)<\delta \text { implies }|W|<\delta .
$$

For a given constant $r>1$, the map $f$ satisfies the backward contracting property with constant $r$, as defined in the introduction, if for every $\delta>0$ sufficiently small the map $f$ is $(\delta, \delta r)$-backward contracting. Given $\delta_{0}>$ 0 and a function $r:\left(0, \delta_{0}\right) \rightarrow(1,+\infty)$, we will say that $f$ is backward contracting with growth function $r$, if for every $\delta \in\left(0, \delta_{0}\right)$ sufficiently small the map $f$ is $(\delta, \delta r(\delta))$-backward contracting.

The purpose of this appendix is to prove the prove the following result.

Theorem 1. For $f \in \mathcal{A}$, the following properties hold.

1. For every $r>1$ there is a contant $K>0$ that depends only on $\ell_{\max }$, such that if $f \in \cup_{N} \mathcal{A}\left(N, \ell_{\max }, K\right)$, then $f$ satisfies the backward contracting property with constant $r$.

2. If $f$ satisfies the summability condition with exponent $\alpha>0$, then there exists $\delta_{0}>0$, and a function $r:\left(0, \delta_{0}\right) \rightarrow(1,+\infty)$ such that for every $\theta \in(0,1)$ we have

$$
\sum_{n \gg 1} r\left(\theta^{n}\right)^{-\alpha}<+\infty
$$

and such that $f$ is backward contracting with growth function $r$.

3. If $f$ satisfies the Collet-Eckmann condition, then there are constants $\alpha \in(0,1]$ and $C>0$, such that $f$ is backward contracting with growth function $r(\delta)=C \delta^{-\alpha}$.

Parts 2 and 3 were proved for rational maps in $[\mathbf{R}$, Theorem A].

For the proof of the theorem, let $f \in \mathcal{A}$. Given $v \in C V$ and $c \in$ Crit let

$$
0 \leq k_{1}(v, c)<k_{2}(v, c)<\cdots
$$

be all integers $k \geq 0$ satisfying the following property: If $r>0$ the smallest number so that the closure of $\hat{B}_{r}(c)$ contains $f^{k}(v)$, then the pull-back of 
the closure of $\hat{B}_{r}(c)$ by $f^{k}$ to $v$ is diffeomorphic. Observe that for certain $v$ and $c$ this sequence might be finite or non-existent. We denote by $\xi_{i}(v, c)$ the corresponding preimage of $c$ by $f^{k_{i}(c, v)}$. Notice that when $\xi_{i}(v, c)$ is close to $v$, the integer $k_{i}(v, c)$ is large.

Let $\eta_{0}=\eta_{0}(f)>0$ be sufficiently small. Then for any $\eta \in\left(0, \eta_{0}\right)$, we have

$$
\left|\hat{B}_{\eta}(c)\right| \approx 2\left(\frac{\eta}{A_{c}}\right)^{1 / \ell_{c}}
$$

and for $x \in \hat{B}_{\eta_{0}}(c)$,

$$
\left|f^{\prime}(x)\right| \approx A_{c} \ell_{c}|x-c|^{\ell_{c}-1},
$$

where $A_{c}=\lim _{y \rightarrow c}|f(y)-f(c)| /|x-c|^{\ell_{c}}$. Here by writing $C_{1} \approx C_{2}$ we mean that $C_{2} / 2 \leq C_{1} \leq 2 C_{2}$.

Lemma 9. There is a constant $C>0$ only depending on $\ell_{\max }$ such that the following property holds. For $\delta>0$ small put

$$
\rho(\delta)=\min \left\{C \cdot A(\delta), \frac{\eta_{0}}{\delta}\right\},
$$

where $A(\delta)$ is equal to

$$
\inf _{\substack{\operatorname{dist}\left(\xi_{i}(v, c), v\right) \geq \delta \\ f_{i}\left(v_{c}\right)+1}(v) \in B_{\eta_{0}}(C V)}\left(\frac{\operatorname{dist}\left(\xi_{i}(v, c), v\right)}{\delta}\left|\left(f^{k_{i}(v, c)+1}\right)^{\prime}(v)\right|\right) .
$$

Then for every $c \in$ Crit, every $n \geq 0$ and every $z \in f^{-n}(c)$ such that for every $i=0, \ldots, n-1$ we have $f^{i}(z) \notin B_{\delta}(C V)$, the pull-back of $\hat{B}_{\delta \rho(\delta)}(c)$ to $z$ by $f^{n}$ is diffeomorphic and disjoint from $C V$.

Proof. Consider a critical point $c \in$ Crit, an integer $n \geq 0$ and $z \in f^{-n}(c)$. Given $\delta>0$ small and $0<r \leq \eta_{0} / \delta$, put $U_{0}=\hat{B}_{\delta r}(c)$ and consider the successive pull-backs $U_{0}, U_{1}, \ldots, U_{n}$, such that $U_{k}$ contains $f^{n-k}(z)$. Let us suppose that for some $k=0, \ldots, n$ the set $U_{k}$ contains a critical value $v \in C V$, and let $k$ be minimal with this property. Note that the restriction of $f^{k}$ to $U_{k}$ is a diffeomorphism onto its image. Moreover, $k=k_{i}(v, c)$ for some $i \geq 1$ and $\xi=\xi_{i}(v, c) \in U_{k}$ is equal to $f^{n-k}(z)$. Then for a constant $C_{1}>0$ only depending on $f$, we have by the One-sided Koebe Principle,

$$
\frac{\operatorname{dist}(\xi, v)}{\left|\hat{B}_{\delta r}(c)\right|} \leq \frac{\operatorname{dist}(\xi, v)}{\operatorname{dist}\left(c, f^{k}(v)\right)} \leq\left(\frac{\operatorname{dist}(\xi, v)}{\operatorname{dist}\left(c, f^{k}(v)\right)}\right)^{\frac{\ell_{c}-1}{\ell_{c}}}\left|\left(f^{k}\right)^{\prime}(v)\right|^{-\frac{1}{\ell_{c}}} C\left(\frac{1}{2}\right)^{\frac{1}{\ell_{c}}}
$$

There is thus a constant $C_{1}>0$ depending only on $\ell_{c}$ such that, if $\delta$ is such that $\delta r$ is sufficiently small, then

$$
\delta r>C_{1} \operatorname{dist}(\xi, v)\left|\left(f^{k+1}\right)^{\prime}(v)\right| .
$$

By hypothesis $\operatorname{dist}(\xi, v) \geq \delta$, so if we take the constant $C$ in the definition of $\rho$ equal to $C_{1}$, then we have $\hat{B}_{\delta \rho(\delta)}(c) \subset U_{0}$ and for each $k=0, \ldots, n$ the set $U_{k}$ is disjoint from $C V$. 
Lemma 10. There is a constant $C_{0}$ that depends only on $\ell_{\max }$ such that the following property holds. For $\delta>0$ put

$$
\begin{aligned}
& r_{0}(\delta)= \\
= & \min \left(31^{-\ell_{\max }} \rho(\delta), C_{0} \inf _{\operatorname{dist}\left(\xi_{i}(v, c), v\right)<\delta}\left(\left(\frac{\delta}{\operatorname{dist}\left(\xi_{i}(v, c), v\right)}\right)^{\ell_{c}-1}\left|\left(f^{k_{i}(v, c)+1}\right)^{\prime}(v)\right|\right)\right) .
\end{aligned}
$$

If for every $\delta>0$ small we have $r_{0}(\delta) \geq 2$, then $f$ is backward contracting with growth function $r_{0}$.

Proof. We will choose the constant $C_{0}>0$ below. Given $c \in$ Crit and $\delta>0$ small, consider successive pull-backs $U_{0}=\hat{B}_{\delta r_{0}(\delta)}(c), U_{1}, \ldots U_{k}$, such that $U_{k} \cap B_{\delta}(C V) \neq \emptyset$. Since for every $\delta>0$ small we have $r_{0}(\delta) \geq 2$, arguing by induction, it is enough to consider the case when for every $i=0, \ldots, k-1$ we have $U_{i} \cap B_{\delta}(C V)=\emptyset$.

For each $i=0, \ldots, k$ let $U_{i}^{\prime}$ and $U_{i}^{\prime \prime}$ be the corresponding pull-backs of $\hat{B}_{10^{\ell \max \delta r_{0}(\delta)}}(c)$ and $\hat{B}_{31^{\ell \max } \delta r_{0}(\delta)}(c)$ respectively, so that $U_{i} \subset U_{i}^{\prime} \subset U_{i}^{\prime \prime}$. Since by definition $31^{\ell_{\max }} r_{0}(\delta) \leq \rho(\delta)$, the previous lemma implies that $f^{k}: U_{k}^{\prime \prime} \rightarrow$ $U_{0}^{\prime \prime}$ is a diffeomorphism. Observe that $U_{0}^{\prime \prime}$ contains a 1-scaled neighborhood of $U_{0}^{\prime}$ and that $U_{0}^{\prime}$ contains a 4-scaled neighborhood of $U_{0}$. In particular, the Koebe Principle implies that the distortion of $f^{k}$ on $U_{k}^{\prime}$ is bounded by 5 .

Case 1. $U_{k}^{\prime}$ is disjoint from $C V$. Since $U_{0}^{\prime}$ contains a 4-scaled neighborhood of $U_{0}$, it follows from the Macroscopic Koebe Principle (Proposition 3 (iv)) that $U_{k}^{\prime}$ contains a 1-scaled neighborhood of $U_{k}$. Since $U_{k} \cap B_{\delta}(C V) \neq \emptyset$ and $U_{k}^{\prime} \cap C V=\emptyset$, it follows that $\left|U_{k}\right|<\delta$.

Case 2. There is $v \in U_{k}^{\prime} \cap C V$. Then there is $i \geq 1$ such that $k=k_{i}(v, c)$ and $\xi=\xi_{i}(v, c) \in U_{k}^{\prime}$ is the unique $k$-th preimage of $c$ in $U_{k}^{\prime}$. Then we must have that $\xi \in B(v, \delta)$; otherwise Lemma 9 would imply that $U_{k}^{\prime \prime} \cap C V=\emptyset$.

Suppose by contradiction that $\left|U_{k}^{\prime}\right| \geq \delta$. Then, if $\delta>0$ is such that $\delta r_{0}(\delta)$ is small, then we have

$$
\begin{aligned}
\frac{\delta}{\left|U_{0}\right|} & \leq 11 \frac{\left|U_{k}^{\prime}\right|}{\left|U_{0}^{\prime}\right|} \leq 55 \frac{\operatorname{dist}(\xi, v)}{\operatorname{dist}\left(c, f^{k}(v)\right)} \\
& \leq 55 \cdot 5^{\frac{1}{\ell_{c}}}\left(\frac{\operatorname{dist}(\xi, v)}{\operatorname{dist}\left(c, f^{k}(v)\right)}\right)^{\frac{\ell_{c}-1}{\ell_{c}}}\left|\left(f^{k}\right)^{\prime}(v)\right|^{-\frac{1}{\ell_{c}}} .
\end{aligned}
$$

So, for a constant $C_{2}>0$ depending only on $\ell_{\max }$, we have

$$
r_{0}(\delta)>C_{2}\left(\frac{\delta}{\operatorname{dist}(\xi, v)}\right)^{\ell_{c}-1}\left|\left(f^{k+1}\right)^{\prime}(v)\right| .
$$

Since $\operatorname{dist}(\xi, v)<\delta$, letting $C_{0}=C_{2}$ we obtain a contradiction.

Proof of Theorem 1. Part 1 is a direct consequence of Lemmas 9 and 10. 
2. Given $\theta \in(0,1)$ we have

$$
\begin{aligned}
& \sum_{n \gg 1} r\left(\theta^{n}\right)^{-\alpha} \leq \\
\leq & C^{\prime} \sum_{i, v, c}\left(\sum_{n \geq 0, \theta^{n}<\operatorname{dist}\left(\xi_{i}(v, c), v\right)}\left(\frac{\operatorname{dist}\left(\xi_{i}(v, c), v\right)}{\theta^{n}}\right)^{-\alpha}\left|\left(f^{k_{i}(v, c)+1}\right)^{\prime}(v)\right|^{-\alpha}+\right. \\
& \left.+\sum_{n \geq 0, \theta^{n}>\operatorname{dist}\left(\xi_{i}(v, c), v\right)}\left(\frac{\theta^{n}}{\operatorname{dist}\left(x_{i}(v, c), v\right)}\right)^{-\alpha\left(\ell_{c}-1\right)}\left|\left(f^{k_{i}(v, c)+1}\right)^{\prime}(v)\right|^{-\alpha}\right) \leq \\
\leq & C^{\prime \prime} \sum_{i, v, c}\left|\left(f^{k_{i}(v, c)+1}\right)^{\prime}(v)\right|^{-\alpha}<+\infty .
\end{aligned}
$$

3. Let $C_{0}>0$ and $\lambda>1$ be such that for every $v \in C V$ we have $\left|\left(f^{k}\right)^{\prime}(v)\right| \geq$ $C_{0} \lambda^{k}$. By [NS1, Theorem B] there are constants $C_{1}>0$ and $\theta \in(0,1)$ such that $\operatorname{dist}\left(\xi_{i}(v, c), v\right) \geq C_{1} \theta^{k_{i}(v, c)}$. Therefore there is $C_{2}>0$ and $\gamma \in(0,1)$ such that,

$$
\left|\left(f^{k_{i}(c, v)+1}\right)^{\prime}(v)\right| \geq C_{0} \lambda^{k_{i}(v, c)} \geq C_{2}\left(\operatorname{dist}\left(\xi_{i}(v, c), v\right)\right)^{-\gamma} .
$$

Choose $\mu \in(0,1)$ and note that, if $\frac{\operatorname{dist}\left(\xi_{i}(v, c), v\right)}{\delta} \leq \delta^{-\mu}$, we have

$$
\left|\left(f^{k_{i}(v, c)+1}\right)^{\prime}(v)\right| \geq C_{2} \operatorname{dist}\left(\xi_{i}(v, c), v\right)^{-\gamma} \geq C_{2} \delta^{-\gamma(1-\mu)} .
$$

Thus there is a constant $C_{4}>0$ such that $r_{0}(\delta) \geq C_{4} \delta^{-\alpha}$, where $\alpha=$ $\min (\mu, \gamma(1-\mu))$.

\section{REFERENCES}

[B1] H. Bruin. The existence of absolutely continuous invariant measures is not a topological invariant for unimodal maps. Ergodic Theory Dynam. Systems 18 (1998), no. 3, 555-565.

[B2] H. Bruin. Invariant measures of interval maps. Ph.D. Thesis. Delft 1994

[BKNS] H. Bruin; G. Keller; T. Nowicki; S. van Strien. Wild attractors exist. Ann. Math. 143 (1996) no. 1, 97-130

[BSS] H. Bruin; W. Shen; S. van Strien. Invariant measure exists without a growth condition. Commun. Math. Phys. 241, 287-306 (2003)

[BS] H. Bruin; S. van Strien. Existence of invariant measures for multimodal interval maps. Global analysis of dynamical systems, 433-447, Inst. Phys., Bristol, 2001.

[CE] P. Collet; J.-P. Eckmann. Positive Liapunov exponents and absolutely continuity for maps of the interval. Ergod. Th. Dyn. Sys. 3, 13-46 (1983)

[GS] J. Graczyk; D. Sands. Manuscript in preparation.

[M] M. Misiurewicz. Absolutely continuous measures for certain maps of an interval. Publ. Math. IHES 53, 17-51 (1981)

[MS] W. de Melo; S. van Strien. One-dimensional dynamics. Springer, 1993

[NS1] T. Nowicki; S. van Strien. Hyperbolicity properties of $C^{2}$ multi-modal ColletEckmann maps without Schwarzian derivative assumptions. Trans. Amer. Math. Soc. 321 (1990), no. 2, 793-810.

[NS2] T. Nowicki; S. van Strien. Invariant measures exist under a summability condition for unimodal maps. Invent. Math. 105, 123-136 (1991)

[R] J. Rivera-Letelier. A connecting lemma for rational maps satisfying a no growth condition. www.arxiv.org/math.DS/0602390.

[SV] S. van Strien; E. Vargas. Real Bounds, ergodicity and negative Schwarzian for multimodal maps J. Amer. Math. Soc. 17 (2004), no. 4, 749-782 


\section{Henk Bruin}

Department of Mathematics,

University of Surrey,

UK

email: h.bruin@surrey.ac.uk

\section{Juan Rivera-Letelier}

Departamento de Matemáticas,

Universidad Católica del Norte,

CHILE

email: rivera-letelier@ucn.cl

\section{Weixiao Shen}

Department of Mathematics,

University of Science and Technology of China,

CHINA P.R.

email: wxshen@ustc.edu.cn

\section{Sebastian van Strien}

Department of Mathematics,

University of Warwick,

UK

email: strien@maths.warwick.ac.uk 